\title{
Mössbauer study of the interface of iron nanocrystallites
}

\author{
J. Balogh, L. Bujdosó, D. Kaptás, and T. Kemény \\ Research Institute for Solid State Physics and Optics, P.O. Box 49, H-1525 Budapest, Hungary \\ I. Vincze \\ Research Institute for Solid State Physics and Optics, P.O. Box 49, H-1525 Budapest, Hungary \\ and General Physics Department, Eötvös University, Budapest, Hungary \\ S. Szabó and D. L. Beke \\ Department of Solid State Physics, Lajos Kossuth University, Debrecen, Hungary
}

(Received 10 August 1998; revised manuscript received 21 June 1999)

\begin{abstract}
The hyperfine parameters of iron atoms are studied in iron nanocrystallites prepared by different methods: ball milling of iron powder, partial crystallization of $\mathrm{Fe}-\mathrm{Zr}-\mathrm{B}-\mathrm{Cu}$ amorphous ribbons, and vacuum evaporation of Fe-B polycrystalline multilayers. Careful analysis of the spectral contribution of the possible impurities and chemical mixing at interfaces reveals that no specific grain boundary contribution can be separated in the Mössbauer spectra when the grain size is in the $2-10 \mathrm{~nm}$ range. The results indicate that excluding chemical effects the hyperfine fields of iron atoms at the bcc interfaces are very close to those in the bulk, and Mössbauer spectra of the iron nanocrystallites studied can be understood without supposing a separate grain boundary phase with very distorted structure or highly reduced density.
\end{abstract}

\section{INTRODUCTION}

It has been assumed for more than a decade that nanocrystalline materials possess a special grain-boundary structure, which is very different from that of the usual polycrystalline grain boundaries. The Gleiter model depicted ${ }^{1}$ nanocrystalline substances as essentially perfect fine grains with wide grain boundaries of significantly reduced density. This way of modeling leads to the very surprising idea that the grainboundary region is disordered to the extent that it lacks even the short-range order of an amorphous or liquid condensed phase. It is described as a gaslike, completely disordered phase. ${ }^{2}$ On the other hand, an increasing amount of structural evidence has been collected recently which confirms that significant structural disorder at the grain boundary extends no further than the planes immediately adjacent to the boundary plane. Besides direct verification by high-resolution transmission electron microscopy, ${ }^{3} \mathrm{x}$-ray absorption fine structure (XAFS) measurements ${ }^{4,5}$ also support that the grain boundary is similar to those in microcrystals. It is also becoming obvious that contamination is a serious problem in most preparation methods; this way, chemical and structural inhomogenities are usually present, ${ }^{6}$ in these materials. In striking contrast to the above-mentioned direct structural evidence, it is still widely accepted that Mössbauer spectroscopy detects the low-density interfacial phase at the grain boundaries.

The structural model discussed above predicts that nanocrystalline materials have a large number of atoms in the grain boundaries which should give a measurable contribution to Mössbauer spectra. In case of 5-10 nm grain diameters, ${ }^{3}$ which is the range of minimum available average grain sizes for most metals, about $30-50 \%$ of the atoms can be found in grain boundaries if a 1-nm grain-boundary thickness is supposed. The first Mössbauer study of nanocrystal- line $\mathrm{Fe}(n-\mathrm{Fe})$ prepared by consolidation of small clusters ${ }^{7}$ was dominantly influenced by the idea of gaslike grainboundary structure. The results were described by two hyperfine components: a sharp sextet with the parameters of pure bcc Fe and a broad sextet with distinct parameters. These components were assigned to atoms inside the crystallites with a nearly perfect order and atoms in the strongly distorted grain-boundary region, respectively. However, later studies revealed ${ }^{8,9}$ large uncertainties in the ratio of the two components and in the hyperfine parameters of the broad component. Following these studies, $n$-Fe particles were prepared by other methods, including chemical ${ }^{10}$ or cluster beam deposition ${ }^{11}$ and mechanical milling. ${ }^{12-16}$ These studies could not reveal an unambiguous common component in the Mössbauer spectra of different $n$-Fe samples which could be safely identified as the one belonging to grain-boundary atoms. On the other hand, the role of different impurities are emphasized in most of these works. ${ }^{10,11,13-15}$

A large variety of the extensively studied soft magnetic nanocrystalline composite materials ${ }^{17}$ also contain $n$-Fe grains besides nanosize amorphous granules. These materials are usually formed by partial crystallization of amorphous ribbons. A spectral component around $30 \mathrm{~T}$ at ambient temperature is often identified ${ }^{18-20}$ as the component belonging to the surface atoms of the bcc Fe precipitates in the amorphous matrix. The possibility of impurity dissolution in bcc $\mathrm{Fe}$ over the equilibrium solubility $\operatorname{limit}^{21}$ has also been raised.

In spite of the contradictions, the concept of a grainboundary contribution in the Mössbauer spectrum of $n$-Fe with broad lines and hyperfine parameters different from the normal $\alpha$-Fe values remained unaltered. ${ }^{22,23}$ Even in a pioneering work for the dynamical properties of nanocrystals a separated grain-boundary phase is considered. ${ }^{24}$ The inconsistency of the data when $n$-Fe samples prepared by different 
methods are compared ${ }^{25}$ is explained by preparation-specific structural differences. The temperature dependence ${ }^{7,8}$ of the so-called grain-boundary and crystalline components was found to be very different. The increased magnetic moment (i.e., the low-temperature hyperfine field) of the grainboundary component was originally explained by a greatly reduced density. About a 20\% reduction of the bulk density ${ }^{7}$ in $n$-Fe samples prepared by the inert gas condensation technique is attributed mainly to a lower density of the grain boundaries, since no voids were found in the compacted powders. The broad lines (i.e., the presence of a distribution of hyperfine fields) and the reduced Curie temperature are explained in terms of exchange fluctuations originating from an amorphouslike, disordered structure. On the other hand, in the $\mathrm{Fe}_{89} \mathrm{Zr}_{7} \mathrm{~B}_{4}$ nanocrystalline composite system the surface layers of the $\mathrm{Fe}$ nanocrystallites were claimed ${ }^{18}$ to show a temperature behavior similar to bcc Fe but the hyperfine field range attributed to it was significantly different, $22-35 \mathrm{~T}$ at $4.2 \mathrm{~K}$. In many later works on $n$-Fe samples prepared by different methods, the observation of a spectral component with a lower hyperfine field at ambient temperature was regarded as an indication of a grain-boundary component without a detailed investigation of its temperature dependence. The supposition of a greatly reduced density and amorphouslike structure clearly contradicts both electronmicroscopy ${ }^{3}$ results on cluster-consolidated nanophase Pd and XAFS (Refs. 4 and 5) measurements on inert gas condensated $\mathrm{Cu}$ and ball-milled $\mathrm{Cu}, \mathrm{Fe}, \mathrm{Ni}$, and $\mathrm{Cr}$.

The aim of the present work is to study $n$-Fe samples prepared by three different methods, to account for the possible impurities, and evaluate the Mössbauer spectra consistently in order to identify a well-defined contribution of the grain-boundary atoms. The following samples will be utilized for this purpose: (a) ball-milled Fe powder, (b) nanocrystalline, i.e., partially crystallized $\mathrm{Fe}-\mathrm{B}-\mathrm{Zr}-\mathrm{Cu}$ amorphous ribbons, and (c) vacuum-evaporated Fe-B multilayers.

Different perturbations are present in the above cases. For ball-milled iron, contamination from the milling toolswhich is mainly $\mathrm{Cr}$ contamination in our case, but $\mathrm{C}, \mathrm{Ni}, \mathrm{W}$, etc., can also be found in certain steels-is to be encountered. In the last two cases, the $n$-Fe grains are embedded in an amorphous matrix. Partial crystallization of amorphous $\mathrm{Fe}-\mathrm{Zr}-\mathrm{B}-\mathrm{Cu}$ results in the appearance of nanosize bcc $\mathrm{Fe}$ crystallites, but as it will be shown these can dissolve $\mathrm{Zr}$ (and B), although the elements are not soluble under equilibrium conditions. In order to eliminate the effects of dissolved impurities in bcc $\mathrm{Fe}$, nanostructured multilayers consisting of insoluble elements are regarded the most relevant. The sample preparation is made under high-vacuum conditions, the level of impurities is the lowest possible, there are no serious porosity problems, and the grain size distribution is narrow. ${ }^{26}$ The grain size is influenced by the columnar growth and is roughly proportional to the layer thickness. ${ }^{27}$ Polycrystalline iron layers with different grain sizes below $10 \mathrm{~nm}$ can be easily produced. Though there are not only bcc-bcc grain boundaries, but other, in our case bccamorphous, interfaces in the sample, a systematic study is able to distinguish them. Multilayers of Fe and B seem to be a good choice since the bulk solubility is less than $10^{-4}(0.01 \%){ }^{28}$ However, in grain boundaries much higher solubility ${ }^{29}$ is expected. According to our results, ${ }^{30}$ an amor- phous Fe-B layer is formed by solid-state amorphization at the interface of the layers and therefore they are also closely related to the samples formed by nanocrystallization of amorphous ribbons. As compared to these samples, it is a fortunate feature of multilayers that during solid-state amorphization B is not dissolved in the bcc phase to a measurable extent. Therefore it is reasonable to state that pure bcc Fe nanocrystals can be studied in these samples. Our data on multilayers will clearly show that the perturbation caused by grain boundaries falls in the range of the experimental linewidth of the Mössbauer spectra. The seeming contradiction between high-resolution electron microscopy and Mössbauer spectroscopy results on the structure of nanocrystalline materials is resolved by attributing the hyperfine component formerly interpreted as the grain-boundary contribution to defect sites and impurities determined by the preparation method.

\section{EXPERIMENT}

The mechanical milling was carried out in a vibrating frame single ball vessel continuously pumped during milling by a conventional diffusion pump system. The typical pressure was $10^{-2} \mathrm{~Pa}$. The technical construction of the vacuum vial corresponds to the description of Ref. 31: a hardened steel ball (60 mm diameter, $870 \mathrm{~g}$ ) oscillates on the top of a 65-mm-diam, 5-mm-thick tungsten carbide bottom plate. The oscillation frequency is $50 \mathrm{~Hz}$; the amplitude of the ball movement is $1.5 \mathrm{~mm}$. For this arrangement a $4 \mathrm{~g}$ powder mixture charge was used to get a high enough alloying rate and low-impurity-concentration. Aldrich Fe powder (99.9\% purity, less than $44 \mu \mathrm{m}$ diameter) was used.

Amorphous $\mathrm{Fe}-\mathrm{Zr}-\mathrm{B}-\mathrm{Cu}$ ribbons and microcrystalline $\mathrm{Fe}_{94} \mathrm{Zr}_{6}$ reference alloy were prepared by rapid quenching from the melt in $\mathrm{H}_{2}$ atmosphere and vacuum, respectively. The nanocrystallizing heat treatment of the amorphous ribbons was carried out in a Perkin Elmer DSC2 calorimeter by heating to the end of the first crystallization peak. The nanocrystalline state of the samples was checked by $\mathrm{x}$-ray diffraction (XRD) and transmission electron microscopy ${ }^{32,33}$ (TEM).

Multilayered samples were evaporated either to Si single crystal or $\mathrm{Al}$ substrates in a vacuum of $10^{-7} \mathrm{~Pa}$ with an evaporation rate of approximately $0.1 \mathrm{~nm} / \mathrm{s}$. The liquidnitrogen-cooled substrate was first covered with up to $30 \mathrm{~nm}$ boron to prevent $\mathrm{Si}$ or $\mathrm{Al}$ contamination, and the topmost layer was 5-nm-thick boron. The layer thickness was controlled by a quartz oscillator, and nominal layer thickness is given using bulk density data. The total sample thickness was between 100 and $200 \mathrm{~nm}$. The polycrystalline multilayers were checked by parallel-beam x-ray diffraction, ${ }^{30}$ transmission electron microscopy, ${ }^{30}$ and neutron reflectometry. ${ }^{34}$ The periodicities determined by these methods were $10-20 \%$ larger than the nominal ones derived from the mass measurements by the quartz oscillator during sample deposition, which can be partly explained by the reduced densities of thin layers. Due to the geometry of the evaporation chamber, there is a variation of the layer thickness throughout the 2-in.-diam samples, which was also checked and was found to be about $0.1 \mathrm{~nm}$. 


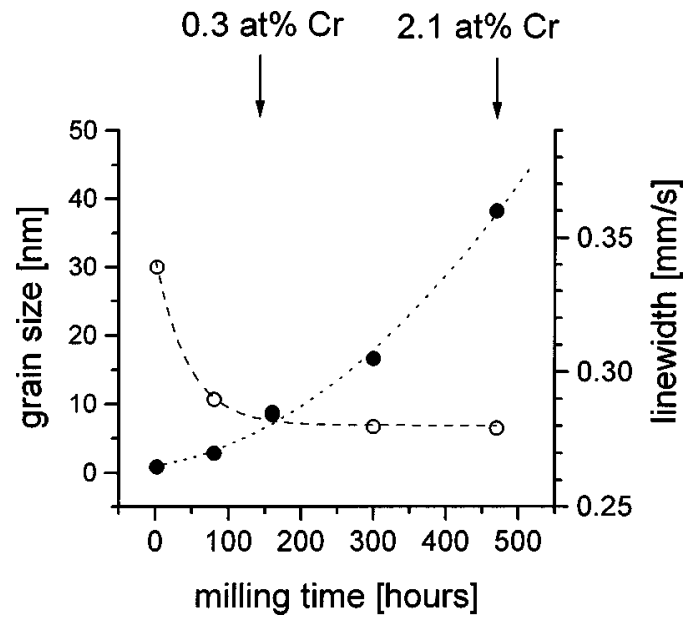

FIG. 1. Grain size determined from the XRD linewidth (open circles and left scale) and the linewidth of the Mössbauer spectrum (solid circles and right scale) of ball-milled Fe powders. The lines are only guide to the eye. $\mathrm{Cr}$ content measured by EDX and x-ray fluorescence is also indicated.

The average grain size of the powder samples was measured by $\mathrm{x}$-ray diffraction of $\mathrm{Cu} K \alpha$ radiation and by transmission electron microscopy (JEOL-2000 FX-II). The impurity content was determined by energy-dispersive $\mathrm{X}$-ray (EDX) and X-ray fluorescence analysis.

${ }^{57} \mathrm{Fe}$ Mössbauer spectra were recorded by a standard constant-acceleration spectrometer using a $50 \mathrm{mCi}{ }^{57} \mathrm{CoRh}$ source. Measurements in applied magnetic field were performed in a Janis cryostat with superconducting magnet.

\section{RESULTS AND DISCUSSION}

\section{A. Ball-milled $n$-Fe samples}

After different milling times the samples were analyzed by XRD to obtain the average grain size and by EDX and $\mathrm{X}$-ray fluorescence to determine the impurity content. The Mössbauer spectra were fitted by one sextet. The observed linewidth as a function of the milling time is plotted in Fig. 1. The average grain sizes, which were determined by the Debye-Scherrer formula from the XRD linewidth, are also shown in the figure. After $100 \mathrm{~h}$ of milling the average grain size determined by XRD was below $10 \mathrm{~nm}$. The grain-size determination can be problematic for two reasons: (i) the Scherrer equation does not take the lattice strain into consideration and, (ii) there might be deviation from the supposed spherical grain shape.

The samples were also examined by transmission electron microscopy ${ }^{35}$ measurements, which provide a more accurate and direct measurement of the grain size. Figure 2 shows a typical dark field image made by the (110)-type reflections of the 160-h-milled sample. The TEM measurements revealed that the grain shapes were elongated and indicated a preferred orientation of the elongated grains in the samples. The thickness of the elongated grains was in accordance with the grain size obtained from the XRD line broadening. The average length per thickness ratio measured over a few hundred grains was over 14 for the $80 \mathrm{~h}$ milling time and were about $10,4.5$, and 1.6 for the milling times 160,300 , and $470 \mathrm{~h}$, respectively. (Elongated bright spots in the dark field image,

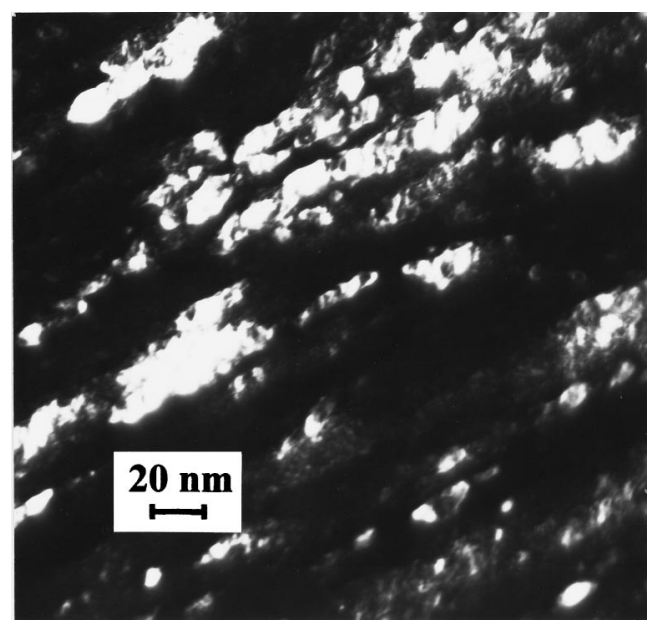

FIG. 2. Dark field TEM image of the 160-h-milled sample.

like those appearing in Fig. 2, were interpreted as single grains and the grayish inner structure was supposed to originate from dislocations lying in bands and/or strained or sheared regions. With this supposition the grain size might be overestimated in some case.) According to the TEM results, the expected grain-boundary fraction approaches $10 \%$ after $160 \mathrm{~h}$ of milling and $25 \%$ after $470 \mathrm{~h}$ of milling if a 1-nm grain-boundary thickness is supposed. The linewidth of the six-line pattern observed in the Mössbauer spectra increased by about $20 \%$ after $160 \mathrm{~h}$ of milling, but $0.3 \% \mathrm{Cr}$ content was also detected by EDX and x-ray fluorescent analysis. After $470 \mathrm{~h}$ milling the $\mathrm{Cr}$ contamination reached 2.1 at. $\%$ and the Mössbauer spectrum also shows features ${ }^{14,36}$ characteristic of $\mathrm{Cr}$ impurities. In order to check the effect of $\mathrm{Cr}$ impurities, both alloy ingots and mechanically alloyed samples were prepared and studied for comparison.

Mössbauer spectra of the Fe powder milled for 160 and $300 \mathrm{~h}$ and that of alloy ingots with $\mathrm{Fe}_{99.7} \mathrm{Cr}_{0.3}$ and $\mathrm{Fe}_{99} \mathrm{Cr}_{1}$ compositions are compared in Fig. 3. Deviations observed in the second and fifth lines are due to sample morphology, which will be discussed later. The two outermost (first and sixth) and the two innermost (third and fourth) lines of the spectra of the 160-h-milled Fe and the $\mathrm{Fe}_{99.7} \mathrm{Cr}_{0.3}$ alloy ingot are identical within the statistical error. There is a small difference in these lines when the sample ball milled for $300 \mathrm{~h}$ and the $\mathrm{Fe}_{99} \mathrm{Cr}_{1}$ ingot are compared: however, this can be due to a $\mathrm{Cr}$ concentration difference less than 0.2 at. \%, which is the experimental error of the measured concentrations. This kind of comparison shows that the gradual increase of the linewidth of the ball-milled Fe samples, as shown in Fig. 1, can be well explained by the measured $\mathrm{Cr}$ impurity content for all of the samples, including the longest milling time.

A further check was made by introducing $\mathrm{Cr}$ impurities into $n$-Fe in a controlled way, i.e., by preparing $\mathrm{Fe}_{100-x} \mathrm{Cr}_{x}$ ball-milled samples with different $(x=0.3,1$, and 5) Cr concentrations. In each case $\mathrm{Fe}$ and $\mathrm{Cr}$ powder mixture and $\mathrm{Fe}_{100-x} \mathrm{Cr}_{x}$ alloy ingots were ball milled in a tube mill working under vacuum for $94 \mathrm{~h}$ to produce small grain-size powder samples. The two kind of samples showed identical Mössbauer spectra and were fitted by a binomial distribution allowing first- and second-neighbor contributions according 


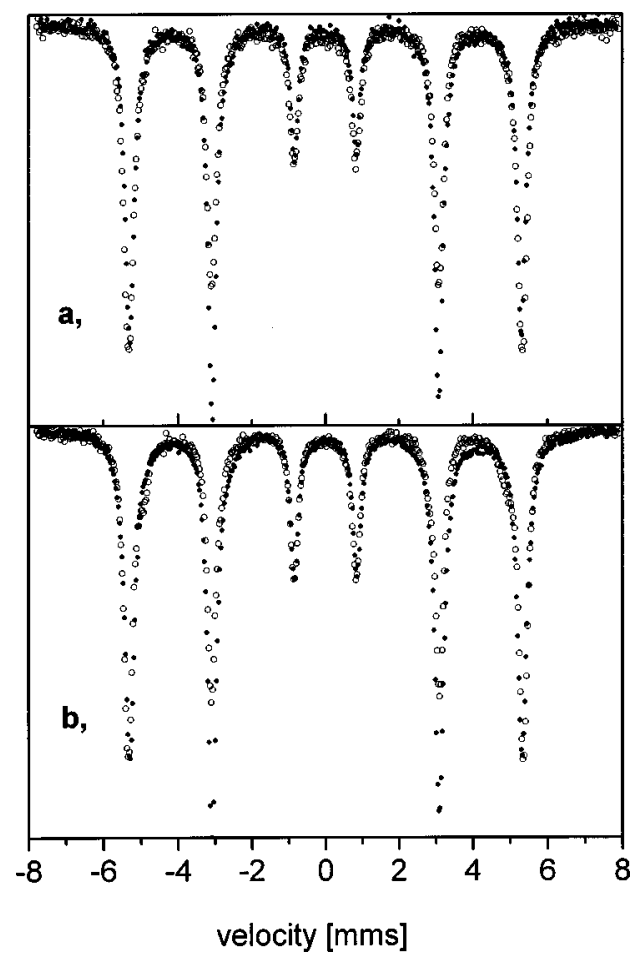

FIG. 3. Mössbauer spectra of Fe powder ball milled (solid circles) for 160 and $300 \mathrm{~h}$ [(a) and (b), respectively] and that of alloy ingots (open circles) with $\mathrm{Fe}_{99.7} \mathrm{Cr}_{0.3}$ and $\mathrm{Fe}_{99} \mathrm{Cr}_{1}$ [(a) and (b), respectively] compositions.

to the bcc structure. This evaluation showed that the $\mathrm{Cr}$ content of the ball-milled samples agrees with the nominal concentration in each case. The milling time $(94 \mathrm{~h})$ is much shorter than the time $(160 \mathrm{~h})$ where significant $\mathrm{Cr}$ contamination could be observed by EDX; i.e., $\mathrm{Cr}$ contamination does not seriously alter the nominal concentration in this case. When the spectra are compared to that of the alloy ingots, ${ }^{37}$ only differences which are in the order of the experimental errors are observed.

Our results for ball-milled $n$-Fe show that no grainboundary contribution can be identified within the experimental limits if the effect of $\mathrm{Cr}$ impurities is taken into account properly. A similar conclusion was drawn ${ }^{38}$ recently by analyzing the results on $n$-Fe prepared by mechanical milling in heptane, where mainly $\mathrm{C}$ impurities have to be encountered. It is also made evident that Mössbauer spectroscopy is a very sensitive tool to detect impurities of the order of 0.1 at. \%. When compared to EDX, which is regarded a sensitive method, ${ }^{39}$ it is to be emphasized that Mössbauer spectroscopy measures a bulk quantity of the sample and therefore it gives a better check of the average impurity concentration when the distribution is not homogeneous. This is obviously the case when wear debris supplies impurities continuously, but an inhomogeneous concentration distribution was also observed when intermixing of $\mathrm{Fe}$ and $\mathrm{Cr}$ powder during ball milling was studied. ${ }^{40}$

There is one significant difference between the spectra of ball-milled $\mathrm{Fe}$ and $\mathrm{Fe}_{100-x} \mathrm{Cr}_{x}$ ingot samples in Fig. 3. The intensity of the second and fifth lines of the spectrum is much greater in case of the ball-milled Fe samples. The difference in the line intensities is a consequence of the different shape of the particles. The ball-milled Fe sample has mostly platelike flakes, while the powdered ingot sample has no significant shape anisotropy. The platelets prefer to lie parallel to the sample plane when the Mössbauer absorber is prepared, and due to the shape anisotropy energy, the magnetic moment of the granules is directed toward the sample plane. This is reflected in the intensity of the second and fifth lines relative to those of the third and fourth lines of the Mössbaur spectrum $\left(I_{2,5}\right)$ according to the formula $I_{2,5}$ $=4 \sin ^{2} \vartheta /\left(1+\cos ^{2} \vartheta\right)$, where $\vartheta$ is the angle between the $\gamma$-ray direction and the magnetic moment. The formation of the platelets might be explained by the plasticity of pure iron at the beginning of the milling. (This is also supported by our results on the ball-milled $\mathrm{Fe}_{100-x} \mathrm{Cr}_{x}$ ingot samples, where this anisotropy was not observed). With the gradual increase of the $\mathrm{Cr}$ content, the $\mathrm{Fe}$ powder becomes harder and more brittle. After prolonged milling the line intensities get again close to $3: 2: 1: 1: 2: 3$, that is, the isotropic average. The increase of the hardness of the powder might explain as well, that the measured impurity content increases more rapidly than a simple linear function of the milling time.

When our Mössbauer spectra are compared to those published by other groups, ${ }^{12,13,15,16}$ the above described change of the line intensities is the only significant difference which can be observed without any fitting procedure. It is connected to the particle shape and might be due to the application of the specific milling equipment. ${ }^{41}$ Otherwise, when the impurity level is low the Mössbauer spectra show only a line broadening similar to all other published results on ballmilled Fe. In accordance with our conclusion this line broadening was attributed to impurities in a few other ball-milling experiments. ${ }^{13,15}$ In other cases the authors claimed that the impurity level was negligible ${ }^{12,16,39}$ and fitted this line broadening by a hyperfine field distribution attributed to a grainboundary phase. However, if we compare these hyperfine field distributions and their spectral fractions, it turns out that the results are quite different from each other. The range of the hyperfine field values seems to depend on the evaluation method applied and the variations of the spectral fractions are not in accordance with the variations of the grain-size values. These inconsistencies may hint at evaluation artifacts when modest line broadening is analyzed by introducing a large number of fitting parameters. It is a well-known problem in Mössbauer spectroscopy, that unphysical oscillation or peaks can emerge in the calculated hyperfine field distributions due to the ill-defined parameter set to be fitted. A large correlation between the fitted parameters indicates that the choice of the parameter set is not correct or the spectrum does not contain enough information to determine them. Some of the commonly used evaluation programs do not warn of the presence of strongly correlated parameters. On the other hand, the shape of the calculated distributions can sensitively depend on the number of sextets, the cutoff hyperfine field values, or the line intensities used in the fitting procedure. This was well demonstrated ${ }^{42}$ by the evaluation of synthetic spectra generated from different hyperfine field distributions of Gaussian shape. Sample thickness effects can cause deviation from the Lorenzian line shape ${ }^{43}$ which may also lead to unphysical peaks when small line broadenings are fitted by a hyperfine field distribution. 


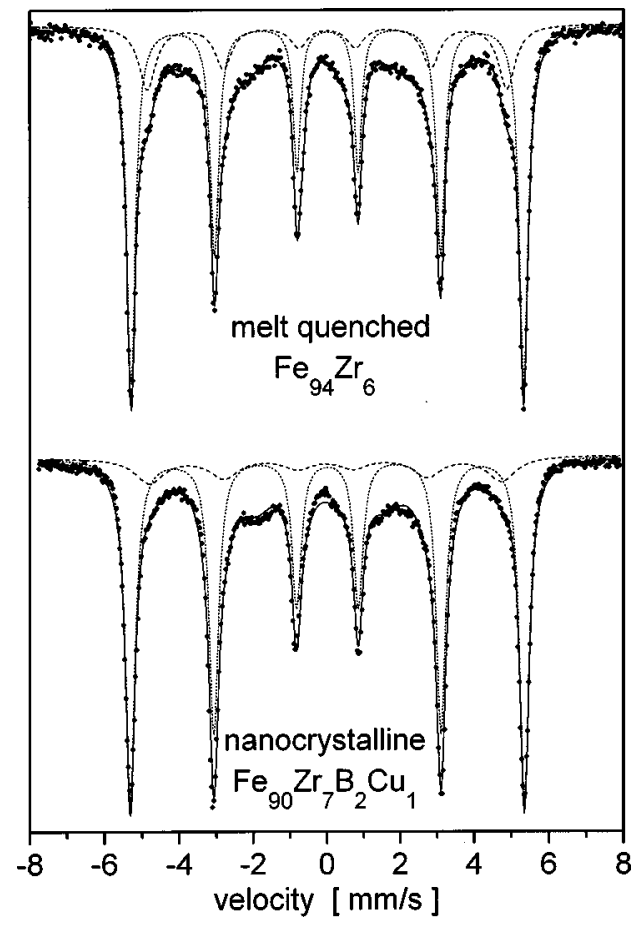

FIG. 4. Room-temperature Mössbauer spectra of the nc $\mathrm{Fe}_{90} \mathrm{Zr}_{7} \mathrm{~B}_{2} \mathrm{Cu}_{1}$ and the nominally $\mathrm{Fe}_{94} \mathrm{Zr}_{6}$ melt-quenched sample. The dotted and dashed curves are the subspectra referred as the main and the satellite components, respectively.

\section{B. Nanocrystallized Fe-Zr-Cu-B amorphous ribbons}

Nanocrystallized (nc) Fe-Zr-B-Cu samples were prepared from amorphous ribbons by heat treatment at a temperature above the first crystallization stage to form bcc nanocrystallites ${ }^{32,33}$ with an average diameter of $10-20 \mathrm{~nm}$ which are embedded in a residual amorphous matrix. Roomtemperature Mössbauer spectra of the $\mathrm{nc} \mathrm{Fe}_{90} \mathrm{Zr}_{7} \mathrm{~B}_{2} \mathrm{Cu}_{1}$ sample are shown in Fig. 4. It contains two well-resolved sextets superimposed on the spectrum of a residual amorphous phase. The first sextet with the larger splitting has hyperfine parameters similar to $\alpha$-Fe, but the origin of the second sextet is disputed. ${ }^{20,21}$ These two components, respectively, will be referred to as the main and satellite components in the followings. The satellite component was usually attributed to $\mathrm{Fe}$ atoms located at the interface of the nanosize $\alpha$-Fe crystallites and the amorphous matrix. However, dissolution of a few at. $\% \mathrm{Zr}$ in the bcc structure may also explain the results. This is not in contradiction with atomic probe field ion microscopy measurements ${ }^{44}$ where no alloying component was observed in the bcc crystallites since the experimental errors of this method cannot exclude a small amount of $\mathrm{Zr}$ and B. Although the equilibrium solid solubility of $\mathrm{Zr}$ in $\mathrm{Fe}$ is negligible, melt quenching offers a possibility for the preparation of the solid solution. The nominal $\mathrm{Fe}_{94} \mathrm{Zr}_{6}$ melt-quenched sample yields a dominant Fe-rich microcrystalline bcc solid solution together with a not-welldefined amorphous or intermetallic minor phase. ${ }^{33}$ The Mössbauer spectrum of this sample together with the main and the satellite subspectra of the bcc phase is shown in Fig. 4. The average zirconium content can be calculated from the intensity ratio of the main and satellite components supposing that the hyperfine field is reduced if a $\mathrm{Fe}$ atom has a $\mathrm{Zr}$

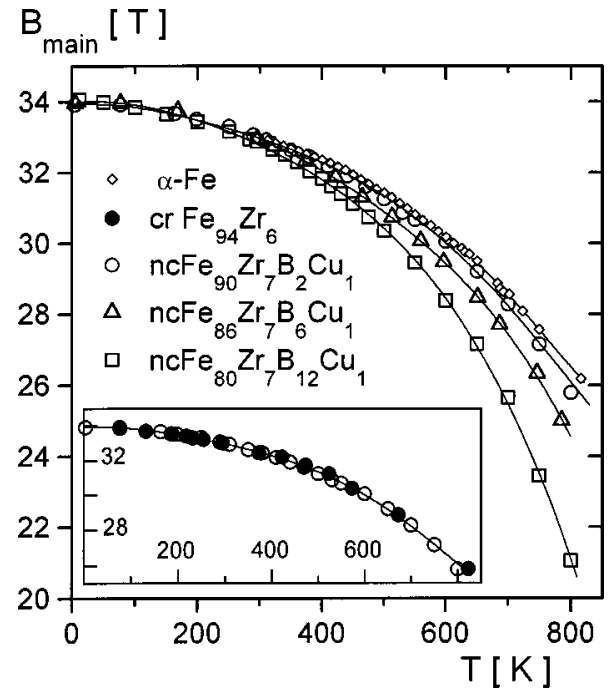

FIG. 5. Temperature dependence of the hyperfine field of the main component $\left(B_{\text {main }}\right)$ of the bcc phase in nc Fe-Zr-B-Cu samples with different metalloid concentrations as compared to a pure $\alpha$-Fe sample. The inset shows the same data points as the main figure for the nc $\mathrm{Fe}_{90} \mathrm{Zr}_{7} \mathrm{~B}_{2} \mathrm{Cu}_{1}$ sample in comparison to the melt quenched nominally $\mathrm{Fe}_{94} \mathrm{Zr}_{6}$ sample.

neighbor in the first two coordination shells. It is 3 at. \%, less than the nominal concentration, and agrees with our estimate for the $\mathrm{nc} \mathrm{Fe}_{90} \mathrm{Zr}_{7} \mathrm{~B}_{2} \mathrm{Cu}_{1}$ sample.

The possibility that the shoulder component of the Mössbauer spectra of nc Fe-Zr-B-Cu samples belong to $\mathrm{Zr}$ (and B) dissolved in the nanosize bcc Fe crystallites is further examined by temperature-dependent studies. The hyperfine field of the main component $\left(B_{\text {main }}\right)$ is shown for nc samples with different metalloid concentrations in Fig. 5. For comparison, data of pure $\alpha$-Fe and the melt-quenched $\mathrm{Fe}_{94} \mathrm{Zr}_{6}$ sample are also shown in the figure. While the low-temperature data agree very well with the pure $\alpha$-Fe value for all of the samples, a significant reduction of the hyperfine fields can be observed at temperatures above $300 \mathrm{~K}$, indicating a reduction of the Curie temperature, which is enhanced by increasing the metalloid content of the sample. The intensity of the satellite component also increases as compared to the intensity of the main line when the metalloid concentration is enhanced. These indicate that $\mathrm{B}$ is also dissolved in the bcc phase and explains the significant reduction of the Curie temperature as the B concentration increases. The inset in Fig. 5 shows that the lowest $\mathrm{B}$ content $\mathrm{nc} \mathrm{Fe}_{90} \mathrm{Zr}_{7} \mathrm{~B}_{2} \mathrm{Cu}_{1}$ and the melt-quenched nominally $\mathrm{Fe}_{94} \mathrm{Zr}_{6}$ sample show similar temperature dependence. It means that a comparable amount of solutes is expected in the bcc phase of these two different samples.

The hyperfine field of the satellite component $\left(B_{\mathrm{sat}}\right)$ follows closely ${ }^{33}$ the temperature dependence of $B_{\text {main }}$ both in the nc and in the melt-quenched samples. $B_{\text {sat }} / B_{\text {main }}$ and $B_{\text {main }} / B_{\mathrm{Fe}}$ (where $B_{\mathrm{Fe}}$ is the hyperfine field of pure $\alpha$-Fe) as a function of temperature is shown for the $\mathrm{nc} \mathrm{Fe}_{80} \mathrm{Zr}_{7} \mathrm{~B}_{12} \mathrm{Cu}_{1}$ sample in Fig. 6. The observation that the hyperfine field of the satellite component $\left(B_{\text {sat }}\right)$ follows closely the temperature dependence of $B_{\text {main }}$-even in case of the nc $\mathrm{Fe}_{80} \mathrm{Zr}_{7} \mathrm{~B}_{12} \mathrm{Cu}_{1}$ sample where the Curie temperature is at least $100 \mathrm{~K}$ lower than the bcc $\mathrm{Fe}$ value-strongly supports 


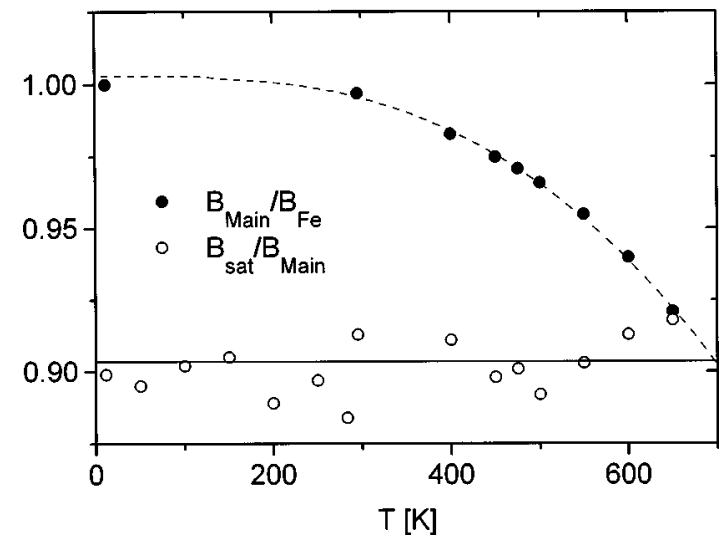

FIG. 6. Hyperfine field ratios of pure $\alpha$-Fe $\left(B_{\mathrm{Fe}}\right)$ and the main $\left(B_{\text {main }}\right)$ and the satellite $\left(B_{\text {sat }}\right)$ components of the bcc phase for nc $\mathrm{Fe}_{80} \mathrm{Zr}_{7} \mathrm{~B}_{12} \mathrm{Cu}_{1}$ as a function of temperature.

the notion that these two components belong to the same phase, i.e., a metastable bcc $\mathrm{Fe}(\mathrm{Zr} / \mathrm{B})$ solid solution. Quite different behavior would be expected if the satellite component were associated with a grain-boundary phase containing the iron atoms on the interface of the bcc nanocrystallites and the amorphous grains, as it was supposed in several papers. ${ }^{18,20}$

\section{Fe-B multilayers}

Room-temperature Mössbauer spectra of samples on $\mathrm{Al}$ foil substrates are shown in Fig. 7. Due to Fe impurities in the $\mathrm{Al}$ substrate, a paramagnetic doublet is encountered which amounts to about 5\% of the total intensity. Besides this, the Mössbauer spectra can be described by two main components. There is a sextet which represents iron atoms in the unperturbed iron layer. It has $32.9 \pm 0.1 \mathrm{~T}$ hyperfine field,

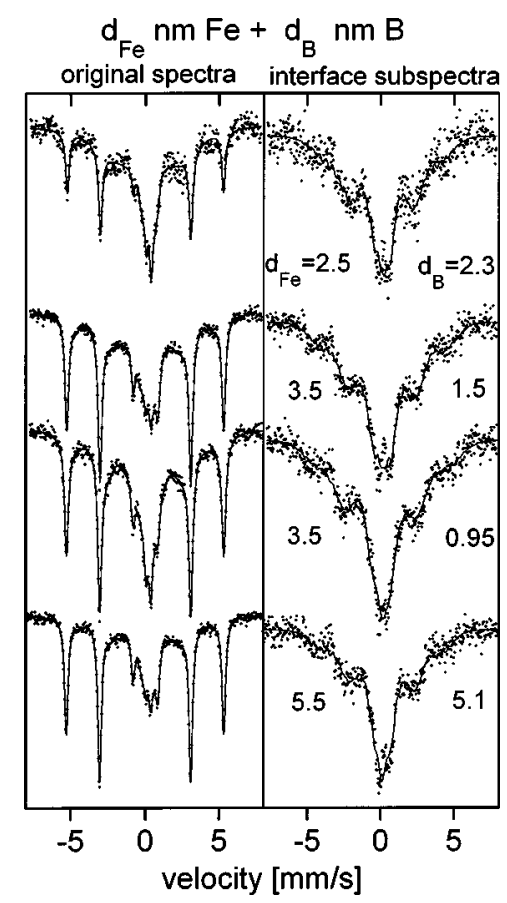

FIG. 7. Room-temperature Mössbauer spectra of samples on $\mathrm{Al}$ foil substrates before (original spectra) and after (interface subspectra) subtraction of the substrate and the pure $\alpha$-Fe contributions. zero isomer shift with respect to pure $\alpha$-Fe, and zero quadruple splitting in case of $d_{\mathrm{Fe}} \geqslant 3.5 \mathrm{~nm}$. (The case of $d_{\mathrm{Fe}}$ $=2.5 \mathrm{~nm}$ is discussed later.) The line intensities within the sextet could be fitted as $3: 4: 1: 1: 4: 3$, showing that the magnetic moments lie in the sample plane. The second component has no sharp lines, but appears as an increased background for larger velocities and gives a peak in the middle of the spectra. This central peak is superimposed to the paramagnetic doublet of the substrate material. A clearer picture of the second component is given when the fitted $\alpha$-Fe sextet and the $\mathrm{Al}$ substrate contribution is subtracted from the measured spectra. These modified spectra (interface subspectra in Fig. 7), attributed to iron atoms in the interface, are very similar for samples on both $\mathrm{Si}$ single crystal $^{30}$ and $\mathrm{Al}$ substrates and for different iron and boron layer thickness values. Considerations concerning the structure and the chemical composition of the interface have been made ${ }^{30}$ by analyzing these figures; however, here our main concern is to show that the interface component can be consistently separated in the spectra. The width of the interface can be estimated from the spectral fraction belonging to it. It is 79 $\pm 5 \%, 57 \pm 5 \%$, and $45 \pm 8 \%$ for the samples with $d_{\mathrm{Fe}}$ $=2.5,3.5$, and $5.5 \mathrm{~nm}$ iron layer thicknesses, respectively. From these values the width of the interface region is estimated to be $2 \pm 0.2 \mathrm{~nm}$ on the iron side, which means that the extent of the bcc iron layer is reduced by this amount due to interface formation on its two sides. This is in perfect agreement with the observation that there is no pure bcc Fe contribution in the Mössbauer spectra ${ }^{30}$ when the iron layer thickness is below $2 \mathrm{~nm}$. After separating the contribution of the Fe-B interface, we turn our attention to the component belonging to the iron layer. The linewidth of the sextet belonging to $\mathrm{Fe}$ atoms in unperturbed iron layer is $0.27 \pm 0.02$ $\mathrm{mm} / \mathrm{s}$ for all samples with $d_{\mathrm{Fe}} \geqslant 3.5 \mathrm{~nm}$ nominal iron layer thickness. Due to interface formation, the actual layer thicknesses are even smaller. Electron microscopy ${ }^{30}$ showed that the individual layers are continuous, their thickness varies less than $1 \mathrm{~nm}$, and a columnar microstructure can be well observed in all the samples. Based on this, the planar grain size can be estimated to scale with the layer thicknesses. After these considerations it can be definitely stated that line broadening or satellite components, as were observed in nanocrystalline iron prepared by other methods, cannot be observed in Fe-B multilayers containing iron grains of similar size.

It is only in case of the $d_{\mathrm{Fe}}=2.5 \mathrm{~nm}$ sample that a significant broadening of the lines $(0.36 \pm 0.02 \mathrm{~mm} / \mathrm{s})$ can be observed. The average thickness of the pure bcc Fe layer is only about $0.5 \mathrm{~nm}$ in this sample, and if we take into account all the experimental uncertainties, it comprises a few atomic layers of bcc Fe. This way the hyperfine parameters of such samples give a good estimate of the order of magnitudes that can be expected in case of grain-boundary atoms. The roomtemperature hyperfine splitting $(32.5 \pm 0.2 \mathrm{~T})$ is slightly reduced compared to the bulk value, but at $4.2 \mathrm{~K}$ they agree within $0.1 \mathrm{~T}$. This probably indicates a significant reduction of the Curie temperature. Mössbauer spectra of this sample at $4.2 \mathrm{~K}$ in an applied field of $3 \mathrm{~T}$ perpendicular to the sample plane are shown in Fig. 8(a). The disappearance of the second and fifth lines of the bcc Fe sextet due to the complete alignment of the magnetic moments can be well observed. 


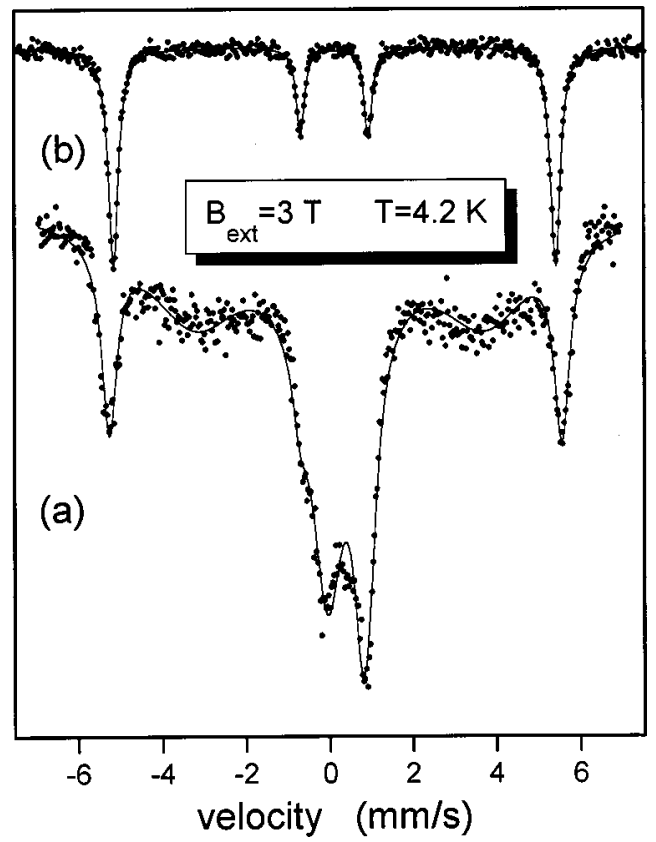

FIG. 8. Mössbauer spectra of the Fe-B multilayer with $d_{\mathrm{Fe}}$ $=2.5 \mathrm{~nm}$ (a) and a 1- $\mu \mathrm{m}$-thick Fe sample evaporated onto a silicon membrane (b) measured at $4.2 \mathrm{~K}$ in a $3 \mathrm{~T}$ magnetic field applied perpendicular to the $\gamma$-ray direction.

On the other hand, the ratio of the bcc Fe and the interfacial component which contains a significant paramagnetic contribution remains unaltered. This further supports that the separation of the interface contribution is correct. The absence of any superparamagnetic relaxation is consistent with the electronmicroscopic observation that the layers are continuous. For comparison, the spectrum of a 1- $\mu$ m-thick Fe sample evaporated onto a silicon membrane and measured under identical conditions is shown in Fig. 8(b). The hyperfine splittings measured in the two samples agree within $0.06 \mathrm{~T}$.

The hyperfine field distribution of the interface ${ }^{30}$ and the calculated interface thickness agree very well in case of different $\mathrm{Fe}$ and B layer thicknesses. Experimental results also exist for other systems with negligible bcc solid solubility [e.g., Fe-Ag (Ref. 45), Fe-Mg (Ref. 46)]. The sophisticated monolayer resolution experiments for $\mathrm{Fe}$ in contact with $\mathrm{Au}$, $\mathrm{Ag}$, and $\mathrm{Cu}$ layers also confirms ${ }^{47}$ that a detectable change of the hyperfine field is restricted to less than three atomic layers and the variations are mainly determined by the chemistry of the interface. The results for systems where the amor- phous interface is paramagnetic at room temperature [e.g., Fe-Zr (Ref. 48), Fe-Ti (Ref. 49] are in acceptable agreement with the observations described above. These experimental results set a limit to the difference between the hyperfine field of iron atoms in the grain-boundary region and in the bulk which is of the order of the experimental linewidth (i.e., below $1 \mathrm{~T}$ ). If this observation is compared to dilute solid solution studies on bcc Fe, one can state that Mössbauer spectroscopy does not support those models which assume a separate grain-boundary phase with distorted structure and significantly reduced density in nanocrystals. The observed spectral component with an increased low-temperature hyperfine field ${ }^{7,8}$ in samples prepared by consolidation of small clusters might be connected to free iron surfaces, i.e., to sample porosity or gas contamination. In more densely packed iron nanocrystallites, our results limiting the change in the iron hyperfine field at the interface to the magnitude of the Mössbauer linewidth are in line with the experimental results ${ }^{50}$ on the saturation magnetization of electroplated nanocrystalline nickel which was also supported by electronic structure calculations. ${ }^{50}$

\section{CONCLUSION}

No well-resolved component of nanocrystalline grain boundaries can be observed in the Mössbauer spectra of ballmilled $\mathrm{Fe}$ and in nanosize iron clusters of $\mathrm{Fe}-\mathrm{Zr}-\mathrm{B}-\mathrm{Cu}$ composite materials after impurity effects have been accounted for properly. This is explained by the results on polycrystalline multilayers which set a low limit to the perturbation which can be expected in the hyperfine field due to changes of the iron coordination numbers and distances in bcc grain boundaries. The observed maximal line broadening which can be attributed to grain-boundary effects in the case of a few atomic layers of $\mathrm{Fe}$ is less than $40 \%$ of the experimental linewidth. This small effect strongly contradicts the view that nanocrystalline materials have a wide disordered grainboundary region of significantly reduced density and well explained by recent findings of direct structural measurements such as high-resolution electron microscopy ${ }^{3}$ and XAFS. ${ }^{4,5}$

\section{ACKNOWLEDGMENT}

This work was supported by the Hungarian Research Fund (OTKA T020624, T022413, and F-019376).
${ }^{1} \mathrm{H}$. Gleiter, in Mechanical Properties and Deformation Behavior of Materials having Ultra-Fine Microstructures, edited by M. Nastasi, D. M. Parkin, and H. Gleiter (Kluwer Academic, Dordrecht, 1993), p. 3.

${ }^{2}$ X. Zhou, R. Birringer, U. Herr, and H. Gleiter, Phys. Rev. B 35, 9085 (1987); T. Haubold, R. Birringer, B. Lengeler, and H. Gleiter, Phys. Lett. A 135, 461 (1989).

${ }^{3}$ R. W. Siegel, in Mechanical Properties and Deformation Behavior of Materials having Ultra-Fine Microstructures (Ref. 1), p. 509.
${ }^{4}$ E. A. Stern, R. W. Siegel, M. Newville, P. G. Sanders, and D. Haskel, Phys. Rev. Lett. 75, 3874 (1995).

${ }^{5}$ J. Schilling, J.-H. He, and E. Ma, J. Phys. IV 7, C2-1221 (1997).

${ }^{6}$ D. G. Morris, Mater. Sci. Forum 269-272, 11 (1998).

${ }^{7}$ U. Herr, J. Jing, R. Birringer, U. Gonser, and H. Gleiter, Appl. Phys. Lett. 50, 472 (1987).

${ }^{8}$ A. Krämer, J. Jing, and U. Gonser, Hyperfine Interact. 54, 591 (1990).

${ }^{9}$ S. J. Campbell, J. Chadwick, R. J. Pollard, H. Gleiter, and U. Gonser, Physica B 205, 72 (1995). 
${ }^{10}$ I. Bødker, S. Mørup, and S. Linderoth, Phys. Rev. Lett. 72, 282 (1994).

${ }^{11}$ J. P. Perez et al., J. Magn. Magn. Mater. 145, 74 (1995).

${ }^{12}$ L. Del Bianco, A. Hernando, E. Bonetti, and E. Navarro, Phys. Rev. B 56, 8894 (1997).

${ }^{13}$ Q. A. Pankhurst, N. S. Cohen, and M. Odlyha, J. Phys.: Condens. Matter 10, 1665 (1998).

${ }^{14}$ J. Balogh, T. Kemény, I. Vincze, S. Szabó, D. L. Beke, and J. Tóth, Phys. Rev. B 59, 14786 (1999).

${ }^{15}$ P. Sinha and G. S. Collins, Hyperfine Interact. 92, 949 (1994).

${ }^{16}$ J. Rawers, D. Cook, and Tae Kim, Philos. Mag. A 78, 965 (1998).

${ }^{17}$ K. Suzuki, Mater. Sci. Forum 312-314, 521 (1999).

${ }^{18}$ A. Slawska-Waniewska and J. M. Greneche, Phys. Rev. B 56, R8491 (1997).

${ }^{19}$ M. Kopcewicz, A. Grabias, and I. Skorvánek, J. Appl. Phys. 83, 935 (1998).

${ }^{20}$ M. Miglierini and J. M. Greneche, J. Phys.: Condens. Matter 9, 2303 (1997); 9, 2321 (1997).

${ }^{21}$ K. Suzuki and J. M. Cadogan, J. Appl. Phys. 85, 4400 (1999).

${ }^{22}$ S. Trapp, C. T. Limbach, U. Gonser, S. J. Campbell, and H. Gleiter, Phys. Rev. Lett. 75, 3760 (1995).

${ }^{23}$ Y. Sasaki, M. Hyakkai, Eiji Kita, A. Tasaki, H. Tanimoto, and Y. Iwamoto, J. Appl. Phys. 81, 4736 (1997).

${ }^{24}$ B. Fultz, C. C. Ahn, E. E. Alp, W. Sturhahn, and T. S. Toellner, Phys. Rev. Lett. 79, 937 (1997).

${ }^{25}$ G. Le Caër and P. Delcroix, Czech. J. Phys. 47, 489 (1997).

${ }^{26}$ C. G. Granqvist and R. H. Buhrman, J. Appl. Phys. 47, 2200 (1976).

${ }^{27}$ Zs. Czigány and G. Radnóczi, Thin Solid Films 347, 133 (1999).

${ }^{28}$ O. Kubaschewski, Iron Binary Phase Diagrams (Springer, Berlin, 1982).

${ }^{29}$ E. D. Hondros and M. P. Seah, in Physical Metallurgy, edited by R. W. Cahn and P. Haasen (Elsevier, Amsterdam, 1983).

${ }^{30}$ J. Balogh, L. Bujdosó, T. Kemény, T. Pusztai, L. Tóth, and I. Vincze, Appl. Phys. A: Mater. Sci. Process. 65, 23 (1997).

${ }^{31}$ L. M. Di, P. I. Loeff, and H. Bakker, J. Less-Common Met. 168, 183 (1991).

${ }^{32}$ T. Kemény, L. K. Varga, L. F. Kiss, J. Balogh, A. Lovas, L. Tóth, and I. Vincze, in Supplement to the Proceedings of the 9th In- ternational Conference on Rapidly Quenched and Metastable Materials, Bratislava, 1996, edited by P. Duhaj, P. Mrafko, and P. Svec (Elsevier, Amsterdam, 1997), p. 201.

${ }^{33}$ T. Kemény, L. K. Varga, L. F. Kiss, J. Balogh, T. Pusztai, L. Tóth, and I. Vincze, Mater. Sci. Forum 226-229, 419 (1998).

${ }^{34}$ R. J. Cooper, J. Balogh, N. Cowlam, and T. Kemény, Mater. Sci. Eng., A 226-228, 90 (1997).

${ }^{35}$ S. Szabó, D. L. Beke, L. Harasztosi, L. Daróczi, Gy. Posgay, and M. Kis-Varga, Nanostruct. Mater. 9, 527 (1997).

${ }^{36}$ S. Szabó, I. Brovko, M. Kis-Varga, D. L. Beke, and Gy. Posgay, Nanostruct. Mater. 6, 973 (1995).

${ }^{37}$ I. Vincze and I. A. Campbell, J. Phys. F: Met. Phys. 3, 647 (1973).

${ }^{38}$ E. P. Elsukov, S. F. Lomaeva, G. N. Konygin, G. A. Dorofeev, V. I. Povstugar, S. S. Mikhailova, A. V. Zagainov, and A. N. Maratkanova, Phys. Met. Metallogr. 87, 114 (1999).

${ }^{39}$ L. Del Bianco, A. Hernando, E. Bonetti, and C. Ballesteros, Phys. Rev. B 59, 14788 (1999).

${ }^{40}$ G. Le Caer, P. Delcroix, T. D. Shen, and B. Malaman, Phys. Rev. B 54, 12775 (1996).

${ }^{41}$ P. Le Brun, E. Gaffet, L. Froyen, and L. Delaey, Scr. Metall. Mater. 26, 1743 (1992).

${ }^{42}$ H. Ren and D. H. Ryan, Phys. Rev. B 51, 15885 (1995).

${ }^{43}$ N. N. Greenwood and T. C. Gibb, Mössbauer Spectroscopy (Chapman and Hall, London, 1971), p. 30.

${ }^{44}$ Y. Zhang, K. Hono, A. Inoue, A. Makino, and T. Sakurai, Acta Mater. 44, 1497 (1996).

${ }^{45}$ U. Herr, J. Jing, U. Gonser, and H. Gleiter, Solid State Commun. 76, 197 (1990).

${ }^{46}$ T. Shinjo, Surf. Sci. Rep. 12, 49 (1991).

${ }^{47}$ M. Przyblski, J. Korecki, W. Karas, and U. Gradmann, Conference Proceedings, ICAME-95, Vol. 50, edited by I. Ortalli (Italian Physical Society, Bologna, 1996), p. 611.

${ }^{48}$ A. Paesano, Jr., S. R. Teixeira, and L. Amaral, J. Appl. Phys. 70, 4870 (1991).

${ }^{49}$ M. Kopcewicz, T. Stobiecki, M. Czapkiewicz, and A. Grabias, J. Phys.: Condens. Matter 9, 103 (1997).

${ }^{50}$ B. Szpunar, U. Erb, G. Palumbo, K. T. Aust, and L. J. Lewis, Phys. Rev. B 53, 5547 (1996). 